\title{
Backbone NMR Assignments of a Prokaryotic Molecular Chaperone, Hsp33 from Escherichia coli
}

\author{
Yoo-Sup Lee and Hyung-Sik Won* \\ Department of Biotechnology, Konkuk University, Chungju, Chungbuk 380-701, Republic of Korea \\ (Received Nov 6, 2012 ; Revised Nov 30, 2012 ; Accepted Dec 10, 2012)
}

\begin{abstract}
The prokaryotic molecular chaperone Hsp33 achieves its holdase activity upon response to oxidative stress particularly at elevated temperature. Despite many structural studies of Hsp33, which were conducted mainly by X-ray crystallography, the actual structures of the Hsp33 in solution remains controversial. Thus, we have initiated NMR study of the reduced, inactive Hsp33 monomer and backbone NMR assignments were obtained in the present study. Based on a series of triple resonance spectra measured on a triply isotope- $\left[{ }^{2} \mathrm{H} /{ }^{13} \mathrm{C} /{ }^{15} \mathrm{~N}\right]$-labeled protein, sequence-specific assignments of the backbone amide signals observed in the 2D$\left[{ }^{1} \mathrm{H} /{ }^{15} \mathrm{~N}\right]$ TROSY spectrum could be completed up to more than $96 \%$. However, even considering the small portion of non-assigned resonances due to the lack of sequential connectivity, we confirmed that the total number of observed signals was quite smaller than that expected from the number of amino acid residues in Hsp33. Thus, it is postulated that peculiar dynamic properties would be involved in the solution structure of the inactive Hsp33 monomer. We expect that the present assignment data would eventually provide the most fundamental and important data for the progressing studies on the 3-dimensional structure and molecular dynamics of Hsp33, which are critical for understanding its activation process.
\end{abstract}

Keywords: Hsp33, oxidative stress, triple resonance, backbone NMR assignments

\section{INTRODUCTION}

The majority of general heat shock proteins function as molecular chaperones under heat-stressed conditions of cells, to prevent degradation of cellular macromolecules. ${ }^{1}$ However, the prokaryotic

Department of Biotechnology, Konkuk University, Chungju, Chungbuk 380-701, Republic of Korea Tel: +82.43.840.3589 Fax:+82.43.852.3616 email: wonhs@kku.ac.kr Journal of the Korean Magnetic Resonance Society 2012 December; 16(2): 172-184 http://dx.doi.org/10.6564/JKMRS.2012.16.2.172 
molecular chaperone Hsp33 is distinctive in that it protects cells from acute oxidative stress. ${ }^{2,3}$ The expression of Hsp33 is also up-regulated by heat at transcriptional level, but the post-translational activation of the protein is dependent on the cellular redox potential. Thus, the activated Hsp33 functions to cope with the oxidative heat condition and/or a severe oxidative stress. Under normal, reducing condition, Hsp33 exists as an inactive, monomeric form that binds a zinc ion via four conserved cysteines at the C-terminal redox-switch domain. It is generally known that the activation process of the inactive Hsp33 monomer is triggered by the oxidation of the conserved cysteines leading to the formation of two intra-molecular disulfide bonds with releasing the zinc ion. Releasing zinc results in the unfolding of the C-terminal, redox-switch domain and finally the protein, as forming dimers and/or oligomers, can achieve a holdase activity that binds folding intermediates of substrate proteins to prevent their ultimate, irreversible denaturation. ${ }^{1-6}$

Several crystal structures of Hsp33 are already available for the Hsp33 from different species and many biochemical and structural studies have been successful in revealing the molecular mechanism of chaperone function. ${ }^{7-11}$ In particular, recent investigations by Reichmann et al. suggested a structural mechanism for the holdase activity of the activated Hsp33 to act on denaturing substrates. ${ }^{12}$ Thus, the Hsp33 has been evaluated as an ideal molecule for in-depth structural analysis of chaperone mechanism. ${ }^{13}$ Nonetheless, a detailed process of the oxidation-induced activation of Hsp33 still remains controversial at the structural level, due to limitations of known structural information, which has been provided mainly by X-ray crystallography. In particular, Hsp33 can adopt several different conformations according to redox status and functional situation, including the reduced monomer, 
oxidized monomer, oxidized dimer, reduced dimer, and oxidized oligomer forms. However, the known crystal structures were not sufficient to elucidate all of the different conformations and contained many doubtful features in physiological relevance. ${ }^{14,15}$ All those uncertainties raised the needs of complementary structural study of Hsp33 by NMR in solution. Thus, in the present study, we report the backbone resonance assignments of the reduced, inactive monomeric form of $\mathrm{Hsp} 33$, as the first step of NMR-based structural investigation.

\section{EXPERIMENTAL METHODS}

The recombinant Hsp33 was prepared from the overproducing E. coli strain BL21(DE3)pLysS containing the plasmid pUJ30 that encodes the Hsp33 from Escherichia coli. ${ }^{2}$ The cells were grown at $37{ }^{\circ} \mathrm{C}$ in $\mathrm{M} 9$ minimal medium, which was prepared with $\mathrm{D}_{2} \mathrm{O}$ and supplemented with $\left[{ }^{15} \mathrm{~N}^{-N_{4}} \mathrm{Cl}\right.$ and $\left[{ }^{13} \mathrm{C}\right]$ glucose, as the sole source of nitrogen and carbon, respectively, to produce the triply isotope $-\left[{ }^{2} \mathrm{H} /{ }^{13} \mathrm{C} /{ }^{15} \mathrm{~N}\right]$-enriched protein. When the $\mathrm{A}_{600}$ of cell growth reached about 0.6 , protein expression was induced by adding IPTG at a final concentration of $1 \mathrm{mM}$. Prior to the induction by IPTG, $1 \mathrm{mM} \mathrm{ZnSO}_{4}$ was added to produce the protein as a zinc-bound form. After 6 hrs induction, cells were harvested by centrifugation and resuspended in a $25 \mathrm{mM}$ Tris- $\mathrm{HCl}$ buffer at $\mathrm{pH}$ 7.5. Additionally, $50 \mu \mathrm{M} \mathrm{ZnSO}_{4}$ and $5 \mathrm{mM}$ dithiothreitol (DTT) was consistently contained in all buffer solutions used, to keep the Hsp33 protein as the inactive monomer form (reduced, zinc-bound form). Cells were disrupted by sonication at $4{ }^{\circ} \mathrm{C}$, and from the supernantant $\mathrm{Hsp} 33$ was purified by 
sequential applications of various chromatographies: two steps of anion exchange chromatography on a HiTrap Q-Sepharose FF and a HiTrap Q HP column (GE Healthcare), adsorption chromatography on a hydroxyapatite column (Bio-Rad), and gel-permeation chromatography on a HiLoad 16/60 Superdex 75 column (Pharmacia). Finally, the purified solution was concentrated to $0.6 \mathrm{mM}$ for NMR measurements in a $20 \mathrm{mM}$ Tris- $\mathrm{HCl}$ buffer (pH 7.4) containing $50 \mathrm{mM} \mathrm{NaCl}, 5 \mathrm{mM}$ DTT, 20 $\mu \mathrm{M} \mathrm{ZnSO}$, and 7\% $\mathrm{D}_{2} \mathrm{O}$. Monomeric state of the purified $\mathrm{Hsp} 33$ was confirmed by the gel-filtration analysis ${ }^{14-17}$ and the concentration was estimated spectrophotometrically, using the molar absorptivity $\left(19,285 \mathrm{M}^{-1} \mathrm{~cm}^{-1}\right.$ at $\left.280 \mathrm{~nm}\right)$ predicted from the amino acid sequence. Conventional 2D$\left[{ }^{1} \mathrm{H} /{ }^{15} \mathrm{~N}\right]$ TROSY and a series of TROSY-based triple resonance spectra $\{\mathrm{HNCA}, \mathrm{HN}(\mathrm{CO}) \mathrm{CA}$, HNCACB, $\mathrm{HN}(\mathrm{CO}) \mathrm{CACB}, \mathrm{HNCO}$ and $\mathrm{HN}(\mathrm{CA}) \mathrm{CO}$ of the $\left[{ }^{13} \mathrm{C} /{ }^{15} \mathrm{~N} /{ }^{2} \mathrm{H}\right] \mathrm{Hsp} 33$ were acquired at 298 K on a Bruker Biospin Avance 900 spectrometer equipped with a cryoprobe. All NMR spectra were processed using NMRPipe/NMRDraw software and analyzed with NMRView program. Chemical shifts were referenced directly to DSS for ${ }^{1} \mathrm{H}$ and indirectly for ${ }^{15} \mathrm{~N}$ and ${ }^{13} \mathrm{C}$ atoms using the chemical shift ratios suggested in the BMRB (http://www.bmrb.wisc.edu).

\section{RESULTS AND DISCUSSION}

Approximately $12 \mathrm{mg}$ of the purified $\left[{ }^{13} \mathrm{C} /{ }^{15} \mathrm{~N} /{ }^{2} \mathrm{H}\right] \mathrm{Hsp} 33$ was finally obtained via $1 \mathrm{~L}$ culture and subjected to NMR measurements. The solution structure of the isolated redox-switch domain (residues 227-287) of Hsp33 has been previously solved by NMR spectroscopy. ${ }^{5}$ Thus, in the present 
study, we employed the same experimental condition for NMR experiments of the intact (residues 1294) Hsp33: $\mathrm{pH} 7.4$ and $298 \mathrm{~K}$. As showing in Fig. 1, the $2 \mathrm{D}-\left[{ }^{1} \mathrm{H} /{ }^{15} \mathrm{~N}\right] \mathrm{TROSY}$ spectrum of the $\left[{ }^{13} \mathrm{C} /{ }^{15} \mathrm{~N} /{ }^{2} \mathrm{H}\right] \mathrm{Hsp} 33$ was obtained with a good dispersion and narrow line widths of amide resonances.

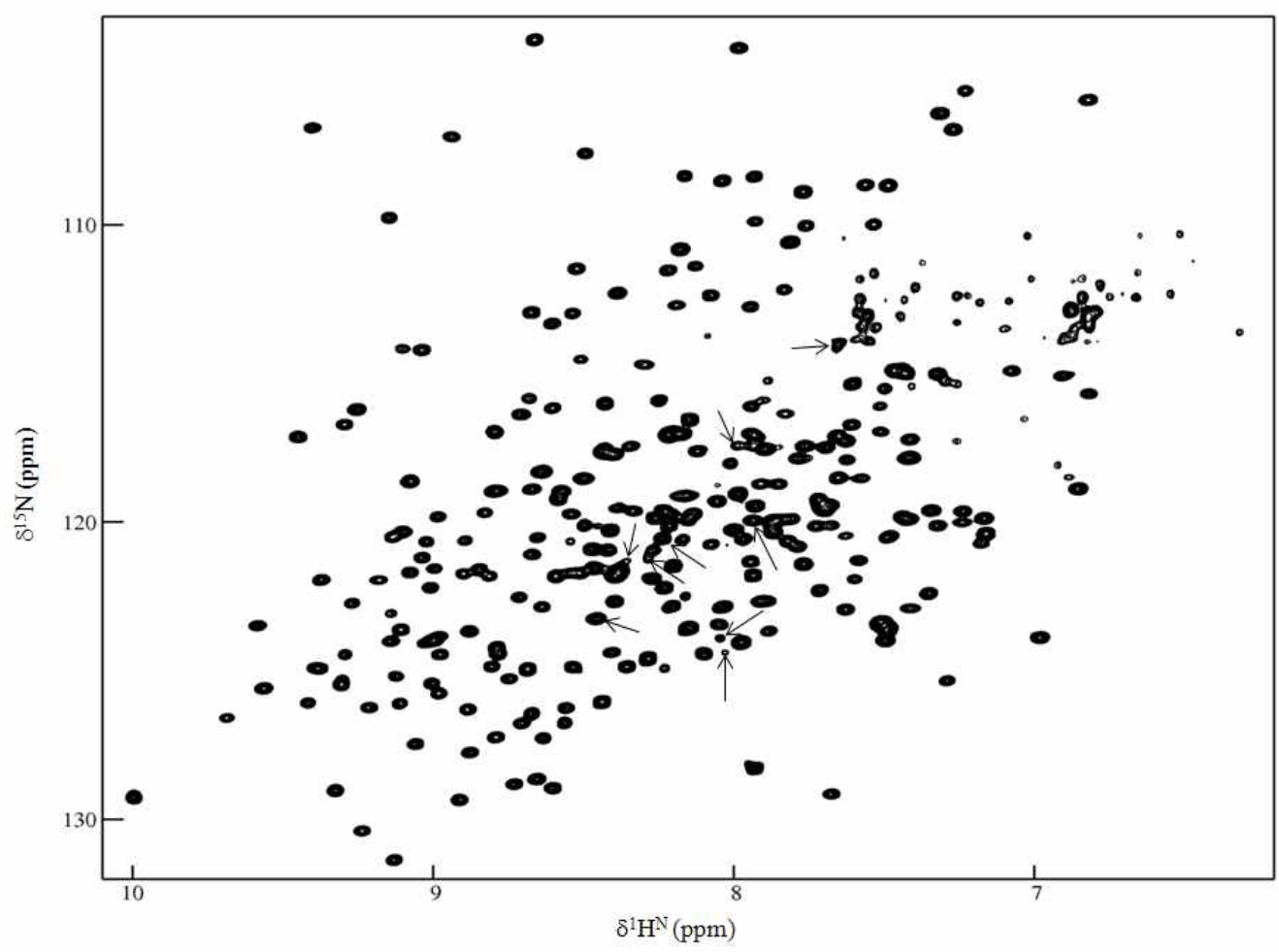

Figure 1. 2D- $\left[{ }^{1} \mathrm{H} /{ }^{15} \mathrm{~N}\right] \mathrm{TROSY}$ spectrum of the $\left[{ }^{13} \mathrm{C} /{ }^{15} \mathrm{~N} /{ }^{2} \mathrm{H}\right] \mathrm{Hsp} 33$ measured on a $900 \mathrm{MHz} \mathrm{NMR}$ machine with a cryoprobe at $298 \mathrm{~K}$. The protein was prepared as a reduced, zinc-bound monomer form at $\mathrm{pH}$ 7.4. Arrows indicate the resonances that were verified as backbone amide signals, but could not be finally assigned due to lack of sequential connectivity. 
For sequence-specific assignments of the backbone amide resonances in the $2 \mathrm{D}-\left[{ }^{1} \mathrm{H} /{ }^{15} \mathrm{~N}\right] \mathrm{TROSY}$ spectrum, we first verified peak clusters of individual resonances by collecting related peaks in the triple resonance spectra.

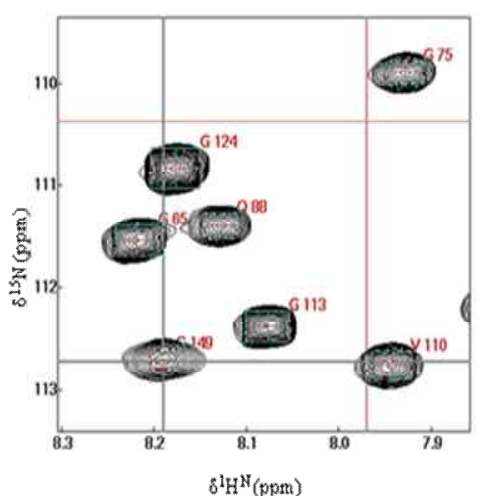

$2 \mathrm{D} \cdot\left[{ }^{1} \mathrm{H},{ }^{15} \mathrm{~N}\right] \mathrm{TROSY}$

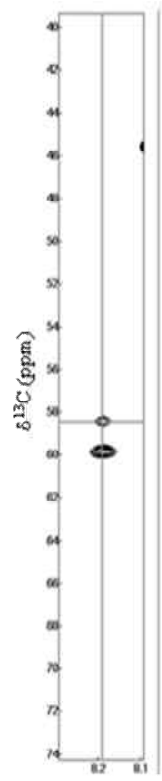

HNCA
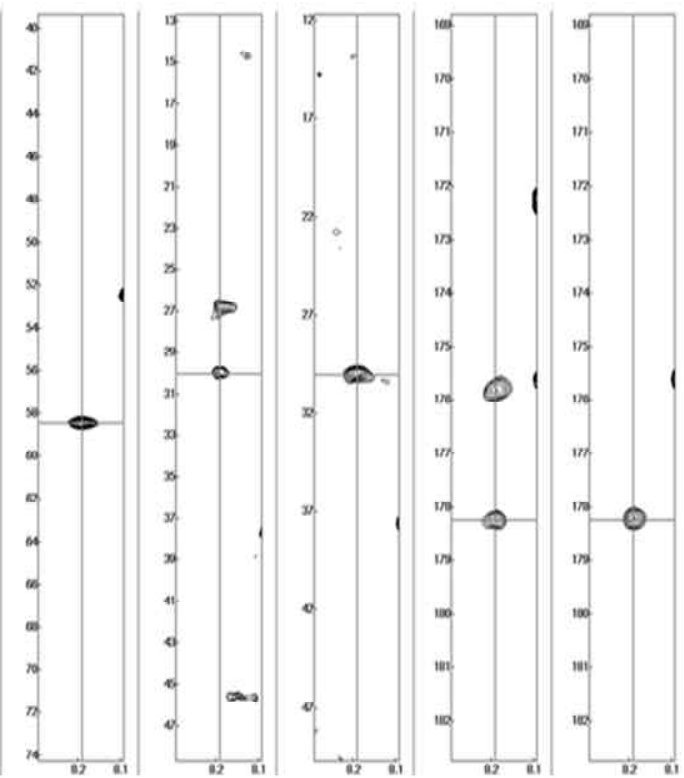

${ }^{2} \mathrm{H}^{\mathrm{N}}(\mathrm{ppm})$

$\mathrm{HN}(\mathrm{CO}) \mathrm{CA} \quad \mathrm{HN}(\mathrm{CA}) \mathrm{CB}$ HN(COCA)CB HN(CA)CO $\mathrm{HNCO}$

Figure 2. Verification of a peak cluster. A selected region taken from the $2 \mathrm{D}-\left[{ }^{1} \mathrm{H} /{ }^{15} \mathrm{~N}\right] \mathrm{TROSY}$ spectrum (Fig. 1) was enlarged in the left panel and the other strip plots for the triple resonance spectra were taken from the slices with the ${ }^{15} \mathrm{~N}$ chemical shift of $112.7 \mathrm{ppm}$. The $\mathrm{x}$-axis of the individual spectrum represents the ${ }^{1} \mathrm{H}$ chemical shift. The y-axes represent the ${ }^{13} \mathrm{C}$ chemical shifts in the triple resonance spectra, while ${ }^{15} \mathrm{~N}$ chemical shifts in the $2 \mathrm{D}-\left[{ }^{1} \mathrm{H} /{ }^{15} \mathrm{~N}\right] \mathrm{TROSY}$ spectrum. The 10 peaks with the same ${ }^{1} \mathrm{H}^{\mathrm{N}}(8.20 \mathrm{ppm})$ and ${ }^{15} \mathrm{~N}(112.7 \mathrm{ppm})$ chemical shift are combined into a peak cluster.

Because all of the backbone NMR signals originating from the same residue manifest the same ${ }^{1} \mathrm{H}^{\mathrm{N}}$ and ${ }^{15} \mathrm{~N}$ chemical shifts in individual spectra, the corresponding peaks could be combined into a 
peak cluster. As illustrated for the S149 residue in Fig. 2, a single peak cluster is composed of ideally 10 peaks with the same amide ${ }^{1} \mathrm{H}^{\mathrm{N}} /{ }^{15} \mathrm{~N}$ chemical shifts in the $2 \mathrm{D}-\left[{ }^{1} \mathrm{H} /{ }^{15} \mathrm{~N}\right] \mathrm{TROSY}$ and six kinds of triple resonance spectra. Thus, each peak cluster additionally contains chemical shift information about the intra- and inter-residue carbon atoms: ${ }^{13} \mathrm{C}^{\alpha}(\mathrm{i})$ and ${ }^{13} \mathrm{C}^{\alpha}(\mathrm{i}-1)$ from the HNCA and $\mathrm{HN}(\mathrm{CO}) \mathrm{CA}$ spectra, ${ }^{13} \mathrm{C}^{\beta}$ (i) and ${ }^{13} \mathrm{C}^{\beta}(\mathrm{i}-1)$ from the $\mathrm{HN}(\mathrm{CA}) \mathrm{CB}$ and $\mathrm{HN}(\mathrm{COCA}) \mathrm{CB}$ spectra, and ${ }^{13} \mathrm{C}^{\prime}(\mathrm{i})$ and ${ }^{13} \mathrm{C}^{\prime}$ (i-1) from the $\mathrm{HN}(\mathrm{CA}) \mathrm{CO}$ and $\mathrm{HNCO}$ spectra. The ${ }^{13} \mathrm{C}^{\alpha},{ }^{13} \mathrm{C}^{\beta}$ and ${ }^{13} \mathrm{C}^{\prime}$ chemical shift values were also useful for prediction of the spin systems of individual clusters. In this way, totally 255 peaks in the $2 \mathrm{D}-\left[{ }^{1} \mathrm{H} /{ }^{15} \mathrm{~N}\right]$ TROSY spectrum was confirmed as backbone amide resonances with reasonable patterns of peak clusters. All the other signals in the $2 \mathrm{D}-\left[{ }^{1} \mathrm{H} /{ }^{15} \mathrm{~N}\right] \mathrm{TROSY}$ spectrum could be also verified unambiguously as side-chain signals from Gln, Asn, Arg, and Trp residues. Then, sequence-specific assignments could be achieved by the sequential linking of the peak clusters, according to the inter-residue ${ }^{13} \mathrm{C}$ connectivities of each cluster. As a representative example, Fig. 3 depicts the sequential, amino-acid specific linking in the region V130 to D136, according to the intraand inter-residue ${ }^{13} \mathrm{C}^{\prime}$ correlations observed in the $\mathrm{HN}(\mathrm{CA}) \mathrm{CO}$ spectrum. Likewise, the ${ }^{13} \mathrm{C}^{\alpha}$ and ${ }^{13} \mathrm{C}^{\beta}$ connectivities were traced in the HNCA and the $\mathrm{HN}(\mathrm{CA}) \mathrm{CB}$ spectrum, respectively (data not shown) The other spectra, $\mathrm{HNCO}, \mathrm{HN}(\mathrm{CO}) \mathrm{CA}$ and $\mathrm{HN}(\mathrm{COCA}) \mathrm{CB}$, were used to confirm the inter-residue ${ }^{13} \mathrm{C}^{\prime},{ }^{13} \mathrm{C}^{\alpha}$ and ${ }^{13} \mathrm{C}^{\beta}$ correlations, respectively. 


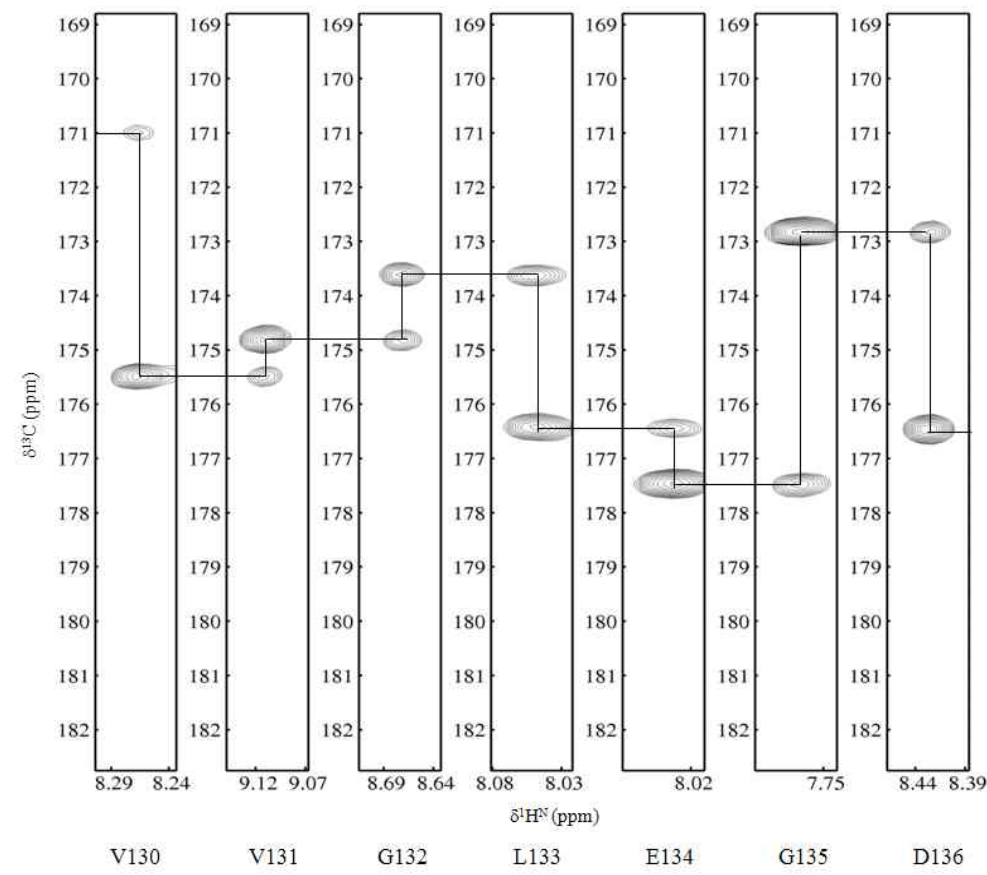

Figure 3. Sequential linking of peak clusters according to the intra- and inter-residue ${ }^{13} \mathrm{C}$ ' correlations. Selected strips from the 3D TROSY-HN(CA)CO spectrum are presented. Solid lines show the connectivities for the region V130 to D136.

Finally, the sequence specific assignments were obtained for total 246 resonances, which correspond to approximately $96.5 \%$ assignments of the observed backbone signals ( 255 peaks) in the 2D- $\left[{ }^{1} \mathrm{H} /{ }^{15} \mathrm{~N}\right]$ TROSY spectrum. The finally assigned backbone amide ${ }^{1} \mathrm{H}^{\mathrm{N}}$ and ${ }^{15} \mathrm{~N}$ chemical shifts are summarized in Table 1. Among the 255 peaks in the $2 \mathrm{D}-\left[{ }^{1} \mathrm{H} /{ }^{15} \mathrm{~N}\right] \mathrm{TROSY}$ spectrum that were identified as backbone amide resonances, just nine signals could not be assigned (Fig. 1) due to the lack of sequential connectivity, despite clear observation of peak clusters. In addition, the results in Table 1 involve a few ambiguous assignments, such as the M3, Y39, Q41 and L57 residues, which 
were determined depending on just ${ }^{13} \mathrm{C}$ chemical shift values without sufficient inter-residue connectivity.

Table 1. ${ }^{1} \mathrm{H}^{\mathrm{N}}$ and ${ }^{15} \mathrm{~N}$ chemical shifts (ppm) assigned in the $2 \mathrm{D}-\left[{ }^{1} \mathrm{H} /{ }^{15} \mathrm{~N}\right] \mathrm{TROSY}$ spectrum of the reduced, zinc-bound Hsp33 at $298 \mathrm{~K}$ and pH 7.4 (NA, not available; ND not detected).

\begin{tabular}{|c|c|c|c|c|c|c|c|c|c|c|c|}
\hline a.a. & ${ }^{1} \mathrm{H}^{N}$ & ${ }^{15} \mathrm{~N}$ & a.a. & ${ }^{\mathrm{T}} \mathrm{H}^{\mathrm{N}}$ & ${ }^{15} \mathrm{~N}$ & a.a. & ${ }^{1} \mathrm{H}^{\mathrm{N}}$ & ${ }^{15} \mathrm{~N}$ & a.a. & ${ }^{1} \mathrm{H}^{\mathrm{N}}$ & ${ }^{15} \mathrm{~N}$ \\
\hline M1 & NA & NA & G75 & 7.94 & 109.9 & S149 & 8.20 & 112.7 & Y223 & 8.37 & 126.4 \\
\hline I2 & NA & NA & P76 & NA & NA & E150 & ND & ND & D224 & 8.51 & 120.1 \\
\hline M3 & 8.45 & 126.1 & M77 & 8.18 & 120.6 & Q151 & 7.45 & 114.8 & P225 & NA & NA \\
\hline P4 & NA & NA & N78 & 7.43 & 117.8 & L152 & 7.21 & 118.9 & Q226 & 8.66 & 120.5 \\
\hline Q5 & ND & ND & L79 & 7.33 & 120.1 & P153 & NA & NA & D227 & 8.68 & 126.4 \\
\hline H6 & ND & ND & A 80 & 8.80 & 127.2 & T154 & 7.80 & 120.8 & V228 & 7.49 & 123.5 \\
\hline D7 & ND & ND & V81 & 9.09 & 121.7 & R155 & 9.40 & 124.9 & E229 & 8.65 & 122.9 \\
\hline Q8 & 7.72 & 119.5 & I82 & 8.42 & 124.4 & L156 & 9.60 & 123.5 & F230 & 8.56 & 121.7 \\
\hline L9 & 9.32 & 125.5 & N83 & 8.80 & 124.4 & F157 & 8.91 & 120.6 & K231 & 7.69 & 129.2 \\
\hline H10 & ND & ND & G84 & 9.16 & 109.8 & I158 & 9.01 & 124.0 & C232 & 8.61 & 129.0 \\
\hline R11 & ND & ND & N85 & 7.33 & 115.0 & R159 & 9.07 & 127.5 & T233 & 8.31 & 114.7 \\
\hline Y12 & ND & ND & N86 & 8.55 & 113.0 & T160 & 8.40 & 112.3 & $\mathrm{C} 234$ & 7.89 & 123.7 \\
\hline L13 & 9.01 & 121.6 & N87 & 7.80 & 117.9 & G161 & 8.05 & 108.5 & S235 & 7.50 & 108.7 \\
\hline F14 & 8.62 & 123.3 & Q88 & 8.14 & 111.4 & D162 & 8.24 & 122.2 & R236 & 9.15 & 124.0 \\
\hline E15 & ND & ND & Q89 & 6.83 & 115.7 & V163 & 8.86 & 121.6 & E237 & 8.65 & 118.3 \\
\hline N16 & 9.11 & 114.2 & M90 & 8.91 & 121.7 & D164 & 9.34 & 129.0 & R218 & 7.88 & 120.4 \\
\hline F17 & 7.09 & 114.9 & R91 & 8.62 & 113.3 & G165 & 8.68 & 103.8 & C239 & 7.70 & 119.6 \\
\hline A18 & ND & ND & G92 & 8.00 & 104.1 & K166 & 7.73 & 122.3 & A240 & 8.47 & 123.2 \\
\hline V19 & ND & ND & V93 & 8.81 & 119.0 & P167 & NA & NA & D241 & 7.67 & 117.1 \\
\hline $\mathrm{R} 20$ & 9.32 & 125.4 & A94 & 7.51 & 124.0 & A168 & 8.22 & 122.8 & A242 & 7.36 & 122.4 \\
\hline G21 & 8.51 & 107.6 & R95 & 9.03 & 120.7 & A169 & 8.17 & 119.1 & L243 & 7.98 & 120.6 \\
\hline E22 & 8.43 & 116.9 & V96 & 8.23 & 120.1 & G170 & 8.95 & 107.0 & K244 & 7.64 & 117.3 \\
\hline L23 & 9.04 & 124.1 & Q97 & 9.39 & 124.9 & G171 & 9.42 & 106.8 & $\mathrm{~T} 245$ & 7.28 & 106.8 \\
\hline V24 & 9.70 & 126.6 & G98 & 7.82 & 110.6 & M172 & 9.31 & 116.7 & L246 & 6.99 & 123.9 \\
\hline T25 & 7.84 & 116.4 & E99 & 8.24 & 119.6 & L173 & 8.99 & 123.9 & P247 & NA & NA \\
\hline V26 & 8.42 & 110.7 & I100 & 8.55 & 124.9 & L174 & 9.14 & 125.2 & D248 & 8.70 & 125.0 \\
\hline S27 & ND & ND & P101 & NA & NA & Q175 & 9.28 & 122.7 & E249 & 9.27 & 116.2 \\
\hline E28 & ND & ND & E102 & 8.48 & 120.9 & Q176 & 7.30 & 125.4 & E250 & 7.18 & 119.9 \\
\hline T29 & ND & ND & N103 & 8.72 & 116.4 & Q177 & 7.90 & 122.7 & V251 & 7.35 & 119.6 \\
\hline L30 & ND & ND & A104 & 7.17 & 120.4 & Q178 & NA & NA & D252 & 8.58 & 119.0 \\
\hline Q31 & 8.35 & 117.4 & D105 & 7.78 & 121.4 & Q179 & 8.5 & 121.5 & S253 & 7.62 & 115.4 \\
\hline Q32 & 7.52 & 117.0 & L106 & 8.22 & 119.8 & Q180 & 7.94 & 117.4 & I254 & 7.64 & 123.0 \\
\hline I33 & 7.64 & 120.5 & K107 & 8.26 & 115.9 & Q181 & 8.44 & 117.6 & L255 & 8.15 & 119.8 \\
\hline L34 & 7.30 & 117.1 & T108 & 8.02 & 118.0 & Q182 & 8.16 & 123.6 & A 256 & 7.96 & 121.4 \\
\hline E35 & 7.44 & 119.9 & L109 & 7.95 & 120.0 & Q183 & 9.19 & 122.0 & E257 & 7.62 & 116.7 \\
\hline N36 & 8.69 & 115.8 & V110 & 7.96 & 112.8 & Q184 & 8.90 & 126.3 & D258 & 8.41 & 117.7 \\
\hline H37 & 7.48 & 114.9 & G111 & 7.77 & 110.0 & Q185 & 8.81 & 117.0 & G259 & 8.18 & 108.4 \\
\hline D38 & ND & ND & N112 & 9.02 & 125.4 & Q186 & 7.25 & 120.0 & E260 & 7.44 & 115.0 \\
\hline Y39 & 8.01 & 120.3 & G113 & 8.09 & 112.4 & Q187 & 7.60 & 121.3 & I261 & 9.05 & 121.2 \\
\hline
\end{tabular}




\begin{tabular}{|c|c|c|c|c|c|c|c|c|c|c|c|}
\hline P40 & NA & NA & Y114 & 8.61 & 116.2 & Q188 & 8.79 & 118.9 & D262 & 8.74 & 128.8 \\
\hline Q41 & 8.14 & 119.6 & V115 & 9.00 & 119.8 & Q189 & 7.83 & 120.6 & M263 & 8.99 & 125.8 \\
\hline P42 & NA & NA & V116 & 9.22 & 126.2 & Q190 & 7.59 & 118.5 & H264 & 8.60 & 121.8 \\
\hline V43 & 6.92 & 115.1 & I117 & 9.14 & 131.4 & Q191 & 8.72 & 126.8 & $\mathrm{C} 265$ & 8.65 & 127.3 \\
\hline K44 & 7.69 & 119.5 & T118 & 9.12 & 123.6 & Q192 & 8.19 & 117.1 & D266 & 8.93 & 129.4 \\
\hline N45 & 7.95 & 116.1 & I1 19 & 9.25 & 130.4 & Q193 & 7.42 & 119.9 & Y267 & 9.15 & 120.5 \\
\hline V46 & 7.25 & 119.6 & T120 & 8.99 & 124.5 & Q194 & 7.53 & 116.1 & C268 & 8.45 & 117.5 \\
\hline L47 & 8.09 & 120.8 & P121 & NA & NA & Q195 & 7.43 & 117.8 & G269 & 7.84 & 112.2 \\
\hline A 48 & 7.82 & 119.9 & S122 & 9.09 & 118.6 & & 6.83 & 105.8 & $\mathrm{~N} 270$ & 8.73 & 122.5 \\
\hline E49 & 7.95 & 117.0 & E1 & 7.94 & & & 7.50 & 123.6 & $\mathrm{H} 271$ & 16 & 116.6 \\
\hline L50 & 8.84 & 119.7 & G1 & 8.19 & 110.8 & $\mathrm{~K} 1$ & 8.67 & 128.7 & Y 272 & 02 & 122.2 \\
\hline L51 & 8.76 & 125.3 & E125 & 8.27 & 119.9 & T199 & 9.15 & 123.1 & L273 & 37 & 124.9 \\
\hline V52 & 7.92 & 118.7 & R126 & 8.41 & 122.7 & E200 & 9.46 & 117.1 & F274 & 9.31 & 124.5 \\
\hline A53 & 8.68 & 118.9 & Y127 & 8.80 & 124.2 & E201 & 6.86 & 118.9 & $\mathrm{~N} 275$ & 9.39 & 121.9 \\
\hline T54 & 8.52 & 114.5 & Q128 & 8.11 & 124.4 & L202 & 7.43 & 117.2 & A 276 & 8.60 & 119.2 \\
\hline S55 & 8.28 & 121.0 & G129 & 9.05 & 114.2 & L203 & 7.57 & 108.7 & M277 & 8.07 & 119.3 \\
\hline L56 & ND & ND & V130 & 8.28 & 121.9 & T204 & 7.24 & 105.5 & D278 & 8.41 & 121.9 \\
\hline L57 & 8.82 & 121.8 & V131 & 9.12 & 126.1 & L205 & 7.52 & 123.4 & I279 & 8.35 & 119.6 \\
\hline T58 & ND & ND & G132 & 8.68 & 113.0 & P206 & NA & NA & A 280 & 7.91 & 122.7 \\
\hline A59 & 6.90 & 118.5 & L133 & 8.06 & 123.5 & A207 & 8.57 & 126.3 & E281 & 7.70 & 117.5 \\
\hline T60 & 7.55 & 110.0 & E134 & 8.05 & 122.9 & N208 & 8.54 & 111.5 & I282 & 7.86 & 119.9 \\
\hline L61 & 7.42 & 122.9 & G135 & 7.78 & 108.9 & E209 & 7.19 & 120.7 & R283 & 8.13 & 117.6 \\
\hline K62 & 8.39 & 121.6 & D136 & 8.44 & 116.0 & $\mathrm{~V} 210$ & 7.61 & 121.9 & $\mathrm{~N} 284$ & 7.91 & 117.6 \\
\hline F63 & 7.50 & 120.5 & T137 & 7.33 & 106.3 & L211 & 7.92 & 115.9 & $\mathrm{~N} 285$ & 8.00 & 119.1 \\
\hline D64 & 8.43 & 120.9 & L138 & 9.58 & 125.6 & W212 & 7.69 & 120.1 & A286 & 7.99 & 124.1 \\
\hline G65 & 8.23 & 111.5 & A139 & 9.11 & 120.3 & $\mathrm{R} 213$ & 8.07 & 118.8 & S287 & 8.23 & 117.1 \\
\hline D66 & 7.73 & 119.3 & A140 & 7.67 & 118.5 & $\mathrm{~L} 214$ & 7.51 & 115.5 & P288 & NA & NA \\
\hline I67 & 8.55 & 119.7 & C141 & 7.78 & 117.4 & Y215 & ND & ND & A289 & 8.30 & 124.6 \\
\hline T68 & 8.89 & 123.7 & L142 & 8.16 & 119.9 & H216 & ND & ND & D291 & 8.21 & 121.5 \\
\hline V69 & 8.89 & 127.8 & E143 & 8.68 & 121.1 & E217 & ND & ND & P291 & NA & NA \\
\hline Q70 & 8.82 & 124.9 & D144 & 7.74 & 120.1 & E218 & ND & ND & Q292 & 8.42 & 120.3 \\
\hline L71 & 9.43 & 126.1 & Y145 & 7.88 & 120.1 & E219 & ND & ND & V293 & 7.95 & 121.8 \\
\hline Q72 & 8.53 & 121.7 & F146 & 8.40 & 119.5 & $\mathrm{~V} 220$ & ND & ND & H294 & 7.94 & 128.3 \\
\hline G73 & 7.94 & 108.4 & M147 & 7.86 & 118.7 & $\mathrm{~T} 221$ & 8.81 & 125.6 & & & \\
\hline D74 & 7.64 & 117.9 & R148 & 8.51 & 118.5 & V222 & 8.57 & 126.8 & & & \\
\hline
\end{tabular}

As Hsp33 is 294 amino acids long, including 14 prolines, total 279 (294 residues minus 14 prolines and the N-terminal residue) backbone amide resonances are expected to be detected in the NMR spectra. Thus, the present results (246 residues assignments) correspond to about $88 \%$ complement of possible amide resonance assignments. However, even including the non-assigned peaks in the $2 \mathrm{D}-\left[{ }^{1} \mathrm{H} /{ }^{15} \mathrm{~N}\right] \mathrm{TROSY}$ spectrum, the number of backbone amide resonances observed $(255$ peaks) are just $91 \%$ of the ideal detection. In other words, it can be estimated that the resonance 
signals from at least about $8 \sim 13 \%(25 \sim 39$ amino acids) residues were not detected in the NMR spectra. We used a highly deuterated protein to overcome the short relaxation time $\left(\mathrm{T}_{2}\right)$ due to long molecular tumbling time, which is expected from the high molecular weight (33 kDa) of Hsp33. Judged from the overall quality (good dispersion and narrow linewidth) of the spectra, which were measured using a ultra-highfield (900 MHz) NMR machine with a cryoprobe, the significant portion of missing signals are not attributable to the high molecular weight of Hsp33. In addition, based on the known crystal structures, the non-assigned regions are not varied in the core of the protein, which rule out the possibility of non-exchangeable amide deuterium of the protein sample. Then, in general, some residues of a protein, particularly at loop regions, may not show up their NMR peaks due to a certain dynamic fluctuation at an intermediate time scale, which results in severe line broadening. However, the non-assigned or non-detected regions contain well-structured elements based on known crystal structures of Hsp33 and are mapped mainly onto the N-terminal regions. Thus, we hypothesize that the non-detected signals would represent a peculiar dynamic motion existing in the reduced, inactive Hsp33 monomer in solution, which is not observed in the crystal structures. To check the possibility, it is required to perform NMR analysis under various experimental conditions with different $\mathrm{pH}$, temperature and magnetic field. In addition, there is no doubt that NMR will be the most appropriate tool to characterize the molecular dynamics of Hsp33 and the present assignment results will provide fundamental information for that analysis. Then, biological significance of the dynamic properties, whether those are critically associated with the chaperone activity and/or functional regulation of Hsp33, is worthy of further investigation. Finally, the present results suggest 
that the Hsp33 structure in solution would include different properties from those observed in the known crystal structures. Thus, we expect that the present result will significantly contribute to progressing investigation of the solution structure of Hsp33.

\section{Acknowledgments}

This work was supported mainly by Basic Science Research Program (no. 2010-0006022) through the National Research Foundation of Korea (NRF) funded by the Ministry of Education, Science and Technology, and in part by the Korea Healthcare Technology R\&D project, Ministry for Health, Welfare \& Family Affairs, Republic of Korea [A092006]. This study made use of the NMR facility at the Korea Basic Science Institute, which is supported by the KBSI high-field NMR research program.

\section{REFERENCES}

1. L. Tutar, Y. Tutar, Curr. Pharm. Biotechnol. 11, 216 (2010).

2. U. Jakob, W. Muse, M. Eser, J.C.A. Bardwell, Cell 96, 341 (1999).

3. J. Winter, M. Ilbert, P.C. Graf, D. Ozcelik, U. Jakob, Cell 135, 691 (2008).

4. M. Ilbert, J. Horst, S. Ahrens, J. Winter, P.C.F. Graf, H. Lilie, U. Jakob, Nat. Struct. Mol. Biol. 14, 556 (2007).

5. H.-S. Won, L.Y. Low, R.D. Guzman, M. Martinez-Yamout, U. Jakob, H.J. Dyson, J. Mol. Biol. 341, 893 (2004).

6. C.M. Cremers, D. Reichmann, J. Hausmann, M. Ilbert, U. Jakob, J. Biol. Chem. 285, 11243 (2010). 
7. I. Janda, Y. Devedjiev, U. Derewenda, Z. Dauter, J. Bielnicki, D.R. Cooper, P.C.F. Graf, A. Joachimiak, U. Jakob, Z.S. Derewenda, Structure 12, 1901 (2004).

8. J. Vijayalakshmi, M.K. Mukhergee, J. Graumann, U. Jakob, M.A. Saper, Structure 9, 367 (2001).

9. S.-J. Kim, D.-G. Jeong, S.-W. Chi, J.-S. Lee, S.-E. Ryu, Nat. Struct. Biol. 8, 459 (2001).

10. L. Jaroszewski, R. Schwarzenbacher, D. McMullan, P. Abdubek, S. Agarwalla, E. Ambing, H. Axelrod, T. Biorac, J.M. Canaves, H.-J. Chiu, et al., Proteins 61, 669 (2005).

11. S-W. Chi, D.G. Jeong, J.R. Woo, H.S. Lee, B.C. Park, B.Y. Kim, R.L. Erikson, S.E. Ryu, S.J. Kim, FEBS Lett. 585, 664 (2011).

12. D. Reichmann, Y. Xu, C.M. Cremers, M. Ilbert, R. Mittelman, M.C. Fitzgerald, U. Jakob, Cell 148, 947 (2012).

13. M.P. Mayer, Cell 148, 843 (2012).

14. Y.-S. Lee. K.-S. Ryu, S.-J. Kim, H.-S. Ko, D.-W. Sim, Y.-H. Jeon, E.-H. Kim, H.-S. Won, FEBS Lett. 586, 411 (2012).

15. Y.-S. Lee, K.-S. Ryu, Y. Lee, S. Kim, K.W. Lee, H.-S. Won, J. Kor. Magn. Reson. Soc. 15, 137 (2011).

16. Y.-S. Lee, H.-S. Ko, K.-S. Ryu, Y.-H. Jeon, H.-S. Won, J. Kor. Magn. Reson. Soc. 14, 117 (2010)

17. D.-W. Sim, Y.-S. Lee, J.-H. Kim, M.-D. Seo, B.-J. Lee, H.-S. Won, BMB Rep. 42, 387 (2009). 